\title{
Sobre los autores
}

\section{Ana Rosas Mantecón}

Doctora en Antropología, Especialista en públicos de cine, museos, turismo y patrimonio, organizaciones que vinculan creatividad e inclusión social, así como en políticas culturales. Ha impulsado el diálogo entre la teoría y la práctica de la gestión cultural a partir de la participación en programas internacionales, nacionales y regionales de profesionalización de gestores, de investigación aplicada y de formación de públicos. Fue coordinadora de un Grupo de Trabajo sobre políticas y consumos culturales del Consejo Latinoamericano de Ciencias Sociales, que agrupó a investigadores de diversos países de América Latina. Su trabajo está radicado actualmente en el Departamento de Antropología de la Universidad Autónoma Metropolitana, México.

\section{Guillermo Hernán Arch}

Arquitecto y estudiante avanzado de la Licenciatura en Filosofía de la Facultad de humanidades y ciencias de la Universidad $\mathrm{Na}$ cional del Litoral Desde 2011 es Presidente y programador de Cine Club Santa Fe y muchos de sus ciclos de Extensión. Ha participado con ponencias en diferentes congresos y encuentros, AFRA 2015, AsAECA 2018 y en los Encuentros de Cine y Filosofía 2017 y 2018 organizados por Cine Club Santa Fe y la cátedra de Estética Facultad de Humanidades y Ciencias de la Universidad Nacional del Litoral y de los cuales formó parte de la organización. Ha sido jurado de los premios Escenario - Diario UNO Santa Fe en la categoría Cine, ediciones 2014 y 2015 y Jurado certamen de video de la Bienal de Arte joven Santa Fe 2010; 2012 y 2014 organizados por la UNL.

\section{Aldana Boragnio}

Doctora en Sociología por la Universidad de Alicante (UA). Magister en Investigación en Ciencias Sociales por la Universidad de Buenos Aires (UBA). Licenciada en Sociología (UBA). Integrante del Grupo de Estudios sobre la Sociología de las Emociones y los Cuerpos del Instituto de Investigaciones Gino Germani y del Centro de Investigaciones y Estudios Sociológicos (CIES). Profesora en la materia Psicología Social de la carrera de Sociología de la Universidad de Buenos Aires. Miembro del equipo editorial de la Revista Latinoamericana de Estudios sobre Cuerpos, Emociones y Sociedad (RELACES). Su trabajo de investigación se centra en las emociones, las prácticas del comer y las comensalidades de mujeres en el ámbito de oficinas públicas en la Ciudad de Buenos Aires.

\section{Celso Sánchez}

Biólogo. Doctor en Educación y profesor asociado en la Universidad Federal del Estado de Río de Janeiro- UNIRIO. Coordinador del Grupo de Estudio de Educación Ambiental desde el Sur, GEASur. 
Patrícia M. Bevilaqua

Artista e investigadora, estudiante de doctorado en Psicosociología de Comunidades y Ecología Social- EICOS- Universidad Federal de Río de Janeiro (UFRJ). Máster en Estudios de Arte Contemporáneo de la Universidad Federal Fluminense (UFF) desde el 2015 y Especialización en Teoría y Praxis del Medio Ambiente por el Instituto de Estudios Religiosos (Iser), 1995. Colaboradora de GEASur.

\section{Bárbara Fortes Campos}

Arquitecto y Urbanista. Máster en Educación de la Universidad Federal del Estado de Río de Janeiro. Investigadora del Grupo de Estudio de Educación Ambiental desde el Sur, GEASur

\section{Bárbara Pelacani}

Bióloga. Máster en Educación por la Universidad Federal del Estado de Río de JaneiroUNIRIO. Estudiante de doctorado en Psicosociología de las Comunidades y Ecología Social en la Universidad Federal de Río de Janeiro (UFRJ). Profesora de la Universidad Federal Rural de Río de Janeiro, miembro del Laboratorio de Memoria, Territorios y Ocupaciones: senderos sensibles, LabMEMS e investigadora del Grupo de Estudios de Educación Ambiental desde el Sur - GEASur.

\section{Rosa García}

Profesora de Historia por la Universidad Nacional del Litoral. Psicóloga Social. Diplomada en Estudios de Género (UNVM). Diplomada en Gestión del Patrimonio Inmaterial (UNCo). Coordinadora del Área Educativa del Museo Etnográfico. Integrante del Programa Institucional Igualdad de Géneros, Derechos y Sexualidades (FHAyCS). Docente de Nivel Superior.

\section{Valentín Magi}

Profesor en Historia por la Universidad Nacional de Rosario. Auxiliar de las cátedras «Historia Argentina l" y "Espacio y Sociedad" en la carrera de Historia de esa misma universidad. Ex becario del Departamento de Estado de los Estados Unidos de América por la Comisión Fulbright.

\section{Facundo Recanati}

Estudiante avanzado de la carrera de Historia de la Universidad Nacional de Rosario. Auxiliar de la cátedra "Espacio y Sociedad".

\section{Javier Miranda}

Licenciado y Profesor en Comunicación Social (UNER) y doctorando en Ciencias Sociales (UNER). Se desempeña como docente en diferentes instituciones de nivel secundario de la ciudad de Paraná, en asignaturas relacionadas con la comunicación, la lengua y la literatura. Es profesor adjunto en la cátedra Sociología de la Educación (FHAyCS-UADER). Reviste como Jefe de Trabajos Prácticos en las asignaturas Sociología de la Educación e Historia de los Medios (FCEDU-UNER). Integra el equipo docente de la asignatura en Historia de los Medios que se dicta en la Licenciatura en Periodismo y Comunicación FHUC-UNL.

\section{Mariana Perticará}

Licenciada en Comunicación Social, Magister en Comunicación y Educación (UAB) y doctoranda en Estudios Sociales (UNL). Se desempeña como profesora adjunta ordinaria en la cátedra Historia de los Medios (FCEDUUNER). Integra el equipo docente del Taller de Planificación y Producción en Comunicación y Educación. Co-dirige la Especialización en Producción de Contenidos y Ambientes Digitales Educativos (FCEDU-UNER), carrera de posgrado en la que dicta regularmente el Seminario "Audiencias, comunidades y 
consumos culturales". Está a cargo del dictado de la asignatura en Historia de los Medios que se dicta en la Licenciatura en Periodismo y Comunicación FHUC-UNL. Actualmente se desempeña como Secretaria de Extensión y Comunicación Institucional de la Facultad de Humanidades y Ciencias de la Universidad Nacional del Litoral.

\section{Roberto Hernández Araya}

Sociólogo y Doctor en Estudios Americanos, especialidad en Pensamiento y Cultura, en el Instituto de Estudios Avanzados de la Universidad de Santiago de Chile (USACH), programa en el cual concluyó su tesis de grado titulada "Pensar el consumo desde América Latina, influencia, discusión y replanteamiento de las teorías de los modos de consumo en la obra de Néstor García Canclini».

Profesor instructor de la Facultad de Administración y Economía de la Universidad de Santiago (FAE-USACH), donde imparte los cursos de "Aspectos sociológicos del consumo", "Teoría sociológica” y "Metodología de investigación". Ha recibido reconocimientos de unidades académicas de distintas universidades chilenas por su desempeño como profesor y tutor de investigación. Asimismo, ha asesorado a instituciones y altas autoridades del ámbito público en su país, donde le ha sido encomendado el apoyo especializado a la toma de decisiones de alcance nacional.

\section{Laila Natalí Pecheny}

Licenciada en Ciencias de la Comunicación de la Facultad de Ciencias Sociales de la Universidad de Buenos Aires (UBA) a partir de la tesina: "Radio Moscú en España (1932-1939). Las intervenciones y sentidos promovidos por el servicio soviético de radiodifusión al exterior durante la crisis social española y la Guerra Civil". Es miembro del equipo de investigación del Proyecto UBACyT "Las aperturas de los 80 en los medios de comunicación y la construcción de un nuevo orden político en Argentina", con sede en el Instituto de Investigaciones Gino Germani (IIGG). También fue parte del comité organizador del Seminario Permanente "Medios, Historia y Sociedad" durante el 2019. Sus trabajos se encuentran en el área de la historia de los medios, la sociología de la cultura y los estudios culturales. Actualmente se encuentra investigando los noticiarios cinematográficos tardíos en la recomposición de los regímenes autoritarios de la década del setenta en España, Argentina y Yugoslavia".

\section{Analía Vanesa Dell' Aquila.}

Licenciada en Historia. Dra en Humanidades y Artes; mención Historia

Es docente de la Facultad de Humanidades y Artes de la Universidad Nacional de Rosario. Profesora Adjunta de la cátedra de Teoría de la Historia de esa Facultad; miembro investigadora del PID «RASTREANDO MEMORIAS: representaciones de Rosario en el imaginario social (18501950)" dirigido por la Dra. Alicia Megías.

Participó de las siguientes publicaciones: Rastreando Memorias. Rosario, Histoira y Representaciones Sociales. 1850-1950. AAVV, UNR Editora; Rosario; 2017; EL Coleccionismo de Arte en Rosario. Colecciones, mercado y exhibiciones, 1880-1970". AA.VV: Fundación Espigas- CEHIPE. Bs. As. 2009. ISBN: 978-987-1398-03-04; Espacio, Memoria e Identidad, UNR Editora; Rosario 2002

Jorge Jofre

Licenciado en Enseñanza de las Artes (UNSAM). Docente terciario y universitario. Participó con ponencias en Jornadas y Congresos. Pública artículos académicos y periodísticos; reseñas y también textos en escritura creativa. Ganó el Primer Premio de Ensayo Cinematográfico (2005.C.C. «Ricardo Rojas”. UBA.) En el 2012 
publicó en Madrid, BERNI X CUATRO TIEMPOS (E.A.E.). Fue columnista (Artes Plásticas / Cine/ Series) de las revistas: Mecenas; Soles; Utopías en la palabra; Punto \& Aparte; Conurbana. cult. y los periódicos: Defensa Informa; El Termómetro. Es Miembro del Círculo de Periodistas de General San Martín (Buenos Aires) y del Grupo Prensa del MNAV (Montevideo).

\section{Elena Rivero}

Magister en Ambiente Construido y Patrimonio Sustentable por la Universidad Federal de Minas Gerais (UFMG), Diplomada en Gestión Cultural por la Universidad Católica de Córdoba (UCC) y Profesora de Historia por la Universidad Nacional del Litoral (UNL). Es Profesora Ayudante de Cátedra en "Sociología" (FBCB-UNL). Es miembro del Centro de Investigaciones en Estudios Culturales, Educativos, Históricos y Comunicacionales radicado en la Facultad de Humanidades y Ciencias (FHUC-UNL). Ha realizado presentaciones en congresos y jornadas, así como publicaciones en revistas académicas con temáticas vinculadas a patrimonio, espacio público, manifestaciones y fotografía.

\section{Sabrina Gil}

Doctora en Letras (UNMDP), Profesora y Licenciada en Historia (UNMDP), Especialista en Lenguajes Artísticos (UNLP), y Profesora de Juegos Dramáticos (UNICEN). Actualmente es becaria postdoctoral del CONICET. Desarrolla su trabajo en el grupo de investigación "Literatura y cultura latinoamericanas" (dirigido por Mónica Marinone y co-dirigido por Gabriela Tineo) radicado en el CELEHIS (Centro de Letras hispanoamericanas) de la Universidad Nacional de Mar del Plata.

\section{Laura Alassia}

Estudiante avanzada Profesorado y Licenciatura en Letras en la Universidad Nacional del Litoral. Becaria entre 2014 a 2016 en el marco del programa BAPI (Beca de Apoyo a los programas institucionales) perteneciente a la Facultad de Humanidades y Ciencias de la Universidad Nacional del Litoral, en la Secretaria de Investigación, realizando tareas concernientes al Programa editorial (difusión y distribución de publicaciones periódicas de la FHUC). Maestra de Inglés para la Enseñanza Media. Desempeño como docente en escuelas de nivel medio desde 2002 hasta 2011. Desde 2017 forma parte del Consejo de Redacción de la Revista Culturas. Debates y perspectivas de un mundo en cambio. Es miembro del Centro de Investigaciones en Estudios Culturales, Educativos, Históricos y Comunicacionales radicado en la Facultad de Humanidades y Ciencias (FHUC-UNL).

\section{Sebastián Moreno Barreneche}

Profesor adjunto en la Facultad de Administración y Ciencias Sociales de la Universidad ORT Uruguay, donde tiene a su cargo los cursos "Cultura y sociedad contemporánea" y "Europa: entre unión y diversidad”, ambos de la Licenciatura en Estudios Internacionales. Licenciado en Filosofía (Universidad de la República, Uruguay) y en Comunicación Social (Universidad Católica del Uruguay). Master of Arts en Filosofía Política, Legal y Económica (Universidad de Berna, Suiza) y Master of Arts en Estudios Globales (Universidad de Graz, Austria). Además de su actividad académica, es asesor en estrategias de comunicación y de marca, con experiencia en organismos internacionales como la Comisión Europea, la ONU y la OTAN.

\section{Raúl Travé Molero}

Docente e investigador en el departamento de Ciencias Sociales y Comunicación de OsteleaEAE-URJC, Escuela Universitaria de Turismo y Hospitalidad. Doctor en Antropología Social y 
Cultural por la Universidad Miguel Hernández de Elche (UMH) es, además, Licenciado en Periodismo por la Universidad Complutense de Madrid y en Antropología Social y Cultural por la UMH. Ha sido investigador invitado en la Universidad de Ljubljana. Sus trabajos de investigación han abordado los procesos de patrimonialización, comunicación y creación de hegemonía en contextos turísticos. Es miembro de los grupos de investigación CULTURDESUMH (Cultura, Turismo y [cooperación al] Desarrollo) e IDITUR-Ostelea (Centro de Investigación, Divulgación e Innovación Turística).

\section{Antonio Miguel Nogués Pedregal} Investigador principal del grupo de investigación CULTURDES en la Universitas Miguel Hernández, donde estudia las relaciones entre cultura, turismo y (cooperación al) desarrollo. Licenciado en Geografía e Historia y Doctor en Antropología Social por la Universidad de Sevilla. Máster en Antropología Cultural por la Universidad de Northwestern (EE.UU.) Ha realizado numerosas estancias en centros de investigación europeos, norteamericanos y latinoamericanos, y ha sido Visiting scholar en las Universidades de Oxford, Lovaina, Ljubljana, Mainz y Wageningen. Autor de Etnografía bajo un espacio turístico (Pasos, 2015), ha coordinado también Culture and society in tourism contexts (Emerald 2012).

\section{Daniel Carmona Zubiri}

Profesor asociado del área de Antropología Social y Cultural del departamento de Ciencias Sociales y Humanidades de la universidad Miguel Hernández de Elche, y Doctor por esta misma universidad desde 2004. Es además Profesor del Cuerpo de Profesores de Enseñanza Secundaria de la especialidad de Geografía e Historia. Su labor en investigación se ha centrado en los procesos de cambio locales de las sociedades tradicionales a las sociedades industriales a través de la cultura material. Asimismo, es miembro del grupo de investigación CULTURDES (Cultura, Turismo y (cooperación al) Desarrollo) y su tarea como investigador aborda cuestiones relacionadas con el patrimonio cultural y el desarrollo turístico local.

\section{Claudia Montoro}

Arquitecta y Profesora Titular Ordinaria de la Cátedra de Historia del Diseño (Introducción a la Historia, Historia I e Historia II) en la Carrea de Licenciatura en Diseño de la Comunicación Visual, de la Facultad de Arquitectura Diseño y Urbanismo de la Universidad del Litoral, desde Junio 2013. Profesora Titular Interina de la Cátedra de Historia del Diseño Industrial de la Carrera de Diseño Industrial (Historia I e Historia II), y Profesora Adjunta Interina de Historia II e Historia III. Cátedra Falco, hoy Collado de la Facultad de Arquitectura, Diseño y Urbanismo de la Universidad Nacional del Litoral. En su formación académica cursó la Maestría en Ciencias Sociales de la FCJS de la UNL y actualmente es Doctoranda del Doctorado en Arquitectura de FADU-UNL. Tesis: "Santa Fe, Ciudad real - Ciudad Imaginada. EI rol de las revistas culturales y técnicas en la construcción de la imagen urbana de modernidad (1908-1946)", con la dirección de la Dra. María del Valle Ledesma. Es miembro del Instituto de Teoría e Historia Urbano- arquitectónica (INTHUAR). Dirigió proyectos de investigación y desarrollo en el marco de los CAI+D convocatorias 2000, 2005, CAI+D 2009 en Red, Nodo 3 y CAI+D 2011 en Red, Nodo 2. Actualmente forma parte del grupo responsable en el Proyecto PE - CAI+D 2016 «Ciudades Universitarias en Argentina en el siglo XX. Una historia comparada, 1905-1983" dirigido por la Dra. Arq. Adriana M. Collado, Facultad de Arquitectura, Diseño y Urbanismo, Universidad Nacional del Litoral. 


\begin{abstract}
Alejandra Fabiana Rodríguez
Profesora adjunta regular e investigadora del Centro Historia Cultura y Memoria de la Universidad Nacional de Quilmes. Es directora del Diplomado de Posgrado en Historia Pública y Divulgación Social de la Historia de la UNQ. Dirigió la Licenciatura en Ciencias Sociales y Humanidades de la misma institución. Magíster en Sociología de la Cultura y Análisis Cultural (UNSaM) y profesora en Enseñanza Media y Superior en Historia (UBA). Actualmente, cursa el Doctorado en Ciencias Sociales (UBA). Se especializa en el campo del cine y la historia y dirige proyectos I+D sobre esta temática. Es autora del libro Historia, pueblos originarios y frontera en el cine nacional (2015), coautora de Un país de película, la historia argentina que el cine nos contó (2009) y compiladora de Tiempo Archivado. Materialidad y espectralidad en el audiovisual (2017), además de haber escrito numerosos artículos y ponencias sobre este campo.
\end{abstract}

